\title{
Automation of Pick and Place Operation in Contact Lens Manufacturing
}

\author{
Jeevaniswaran Thangaraj" and Norhaliza Abdul Wahab
}

School of Electrical Engineering, Faculty of Engineering, Universiti Teknologi Malaysia, Johor Bahru, Malaysia

*Corresponding author: jeevaniswaran2@gmail.com

\begin{abstract}
This paper describes the design of an automation of pick and place operation in contact lens manufacturing. The contact lens pick and place system is designed so that it replaces operators to pick and place contact lenses from cassette to PS-48 tray automatically. The microcontroller used in this project is Arduino Uno. Solidwork is a software that used to create the design before fabrication. Actuators used in this projects are stepper motors due to their high precision in calculating steps to move the overall system. C programming is used along with the Arduino Integrated Development Environment, IDE in programming the movement of the system. In this work, the robot is controlled by pressing start and stop button. The system manages to transfer the lenses from cassette to tray automatically. The automated lens pick and place system is capable of reducing cycle time to complete the process by picking 7 lenses simultaneously. Moreover, the designed lens pick and place robot can reduce the number of labors and improve the hygiene in contact lenses production.
\end{abstract}

Keywords: cassette, contact lenses, vacuum sucker, PS-48 Tray.

Article History: received 7 May 2018; accepted 15 August 2018; published 29 August 2018.

(C) 2018 Penerbit UTM Press. All rights reserved

\section{INTRODUCTION}

In manufacturing industries, human labors are employed to do repetitive task whereby it needs the very high rate of precision and accuracy. Human labors tend to do mistakes and it is unavoidable. In addition, productivity is also affected due to human labors since they have limited working speed and need to take rest. As an alternative, the repetitive task is being automated to overcome the human inefficiency.

Automation as a technology is concerned with the use of mechanical, electrical, electronic and computer-based control systems to replace human beings with machines, not only for physical work but also for the development of information processing [1-3]. Industrial automation is designed and built to improve productivity, product quality and precision in performance. An industrial robot is also an automation whereby it is built in various designs according to the requirements of the task. Labor cost, shortage of workers and errors can be easily solved by automating a repetitive task.

Alcon is an American global medical company specializing in eye care products. Alcon is a Novartis company. They are one of the leading contact lenses manufactures in this world. One of the Alcon branches is located at Tanjung Pelapas Port in Johor. Contact lenses are considered as medical devices and can be worn to correct vision, cosmetic or therapeutic reasons [4][5]. Figures 1 and 2 show the overall contact lens production process flow in Alcon Johor and the process flow of contact lens production at the finishing site and the process being automated.

In Alcon Johor, workers are assigned to pick lens from the cassette and place in PS-48 Tray using vacuum sucking pen manually on by one after the coating process at Linear Plasma machine. Each cassette and tray contain 96 lenses and 48 lenses respectively. One operator is assigned to remove the cassette from the Linear Plasma machine and three more operators to unload the lenses from the cassette. The cycle time to complete a tray is five minutes.

Alcon Johor planned to automate the lens pick and place operation from cassette to PS-48 Tray to reduce labors and cycle time. An automation system can perform pick and place faster with higher precision without stopping. An automation system is designed to transfer the lenses conveniently without any deformation. The design and structure of industrial robots are designed according to the function and complexity to perform the task. The main aim of this project is to automate the contact lens pick and place operation.

\section{RESEARCH METHODOLOGY}

\subsection{Robot Design}

The robot is designed to perform pick and place operation. The robot will pick 14 contact lenses at a time from cassette to PS-48 Tray. Thus, a simple structure with the $\mathrm{X}$ and $\mathrm{Z}$ axis linear movement is sufficient. The design has a total of two axes of movement. The vacuum sucking gripper move front and back to pick and place the contact lenses from cassette to tray.

Meanwhile, the tray holder is capable of holding two trays and it moves up and down from the 1st level of a cassette to the 7th level. For motion and movements of the linear axis, the stepper motor will be acting as the actuators. The proximity sensor is fixed for safety purposes. The machine will stop when a contact occurs, 
developed for human safety in an industrial environment. Figure 3 shows the conceptual design of the lens pick and place robot. This machine needs to design the custom made of nozzle head which contains multiple vacuum sucker and control by pump motor in order to make vacuum sucker operate in same time. So, this machine can multiple picks and place contact lenses in one time with the same amount of pressure. In the same time, this method will reduce the time process of the pick-and-place process compare to manually pick-and-place process.

The estimation time for this machine reducing is 50 percent from manually time process. Figure 4 and Figure 5 show the vacuum sucker and cassette, respectively currently used in the production of ALCON Malaysia. This vacuum operated by touching the sensor to control in and out of pressure from the pneumatic pump.

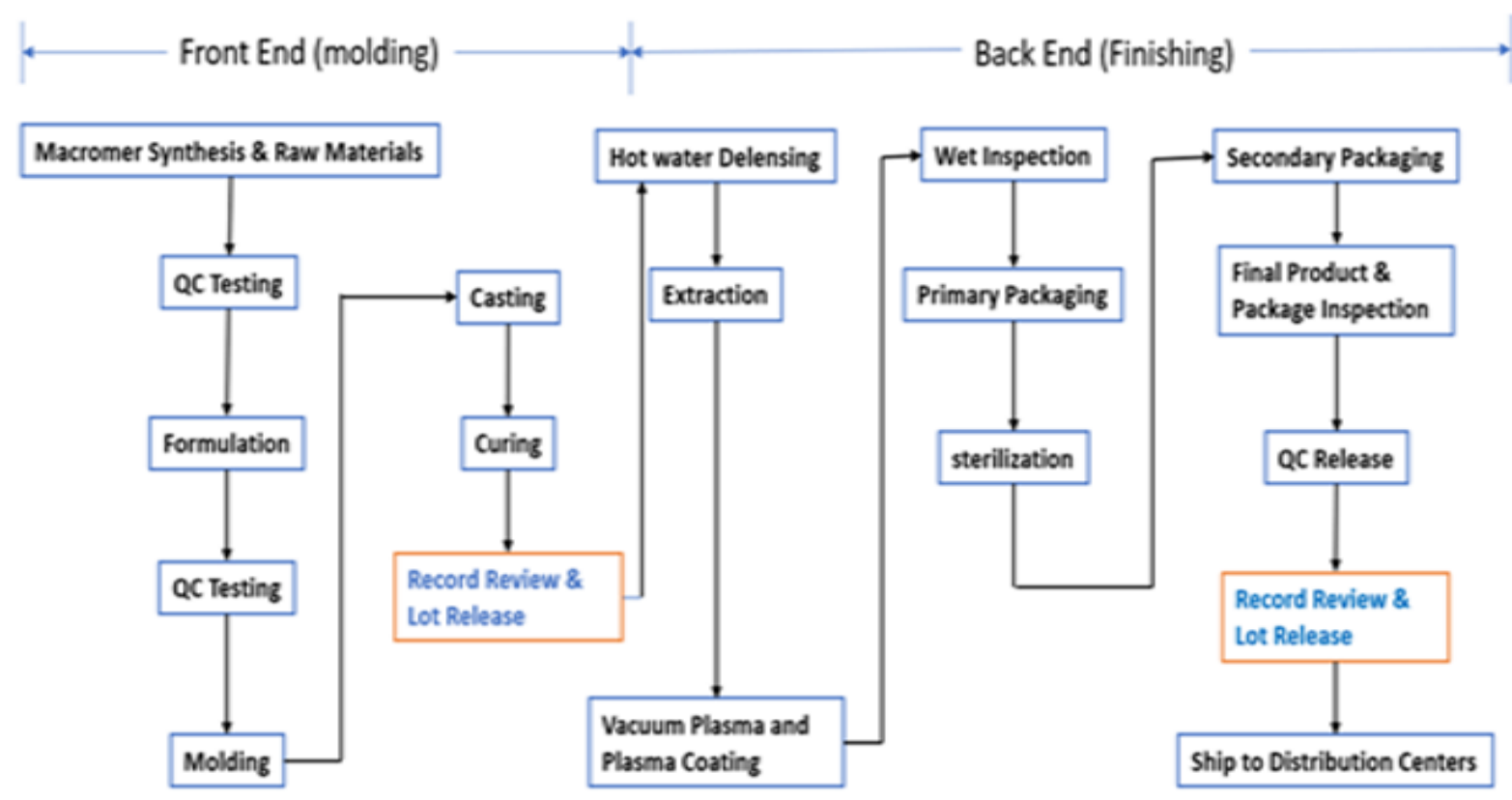

Figure 1. Overall contact lens production process flow in Alcon Johor

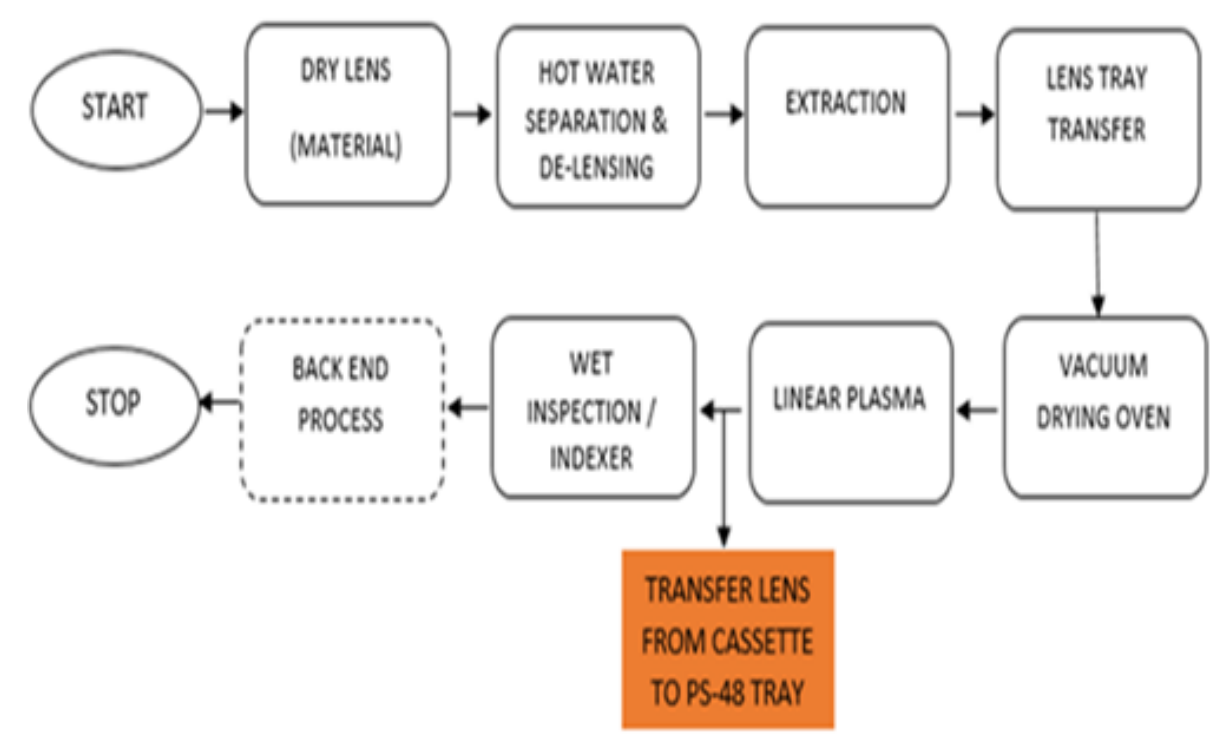

Figure 2. The process flow in contact lens production at finishing site and the process being automated

\subsection{Electrical Development}

In an automatic system, a controller is needed to program with the sensor and mechanism in order to make the machine work properly [6-8]. Nowadays, there is a lot of controllers are used such as PLC, Arduino, PIC microcontroller, Raspberry Pi, ATMEL.

All these controllers actually have same control function but they are different in specification, programming, and design. For this machine, after completing the structure based, the electronics board can be design with space available or specified space. Custom development board can be made to ensure the connection and wiring can be made with less error. This part will show the major electronics component used in this project such as Arduino Uno, limit switch, Stepper motor Nema 17 with TB6560 and L298N motor driver and power 
supply (12V \& 10 A supply) [9][10].

The advantages of using stepper motor are faster positioning time during short strokes, overload saves, high torque in high speed and simplicity of construction [11-14]. Besides that, the advantages of using Arduino Uno very easy to extend it and has tons of usercontributed shields and libraries [15][16]. Shields are available to do pretty much anything, can be used in realtime applications, many input \& output and open source (both hardware, software and IDE).

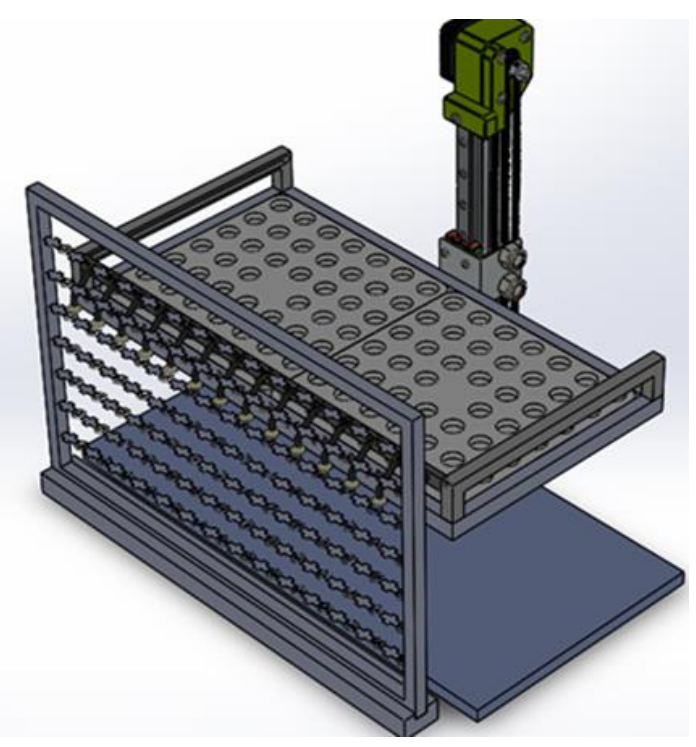

Figure 3. Design of the Automation System

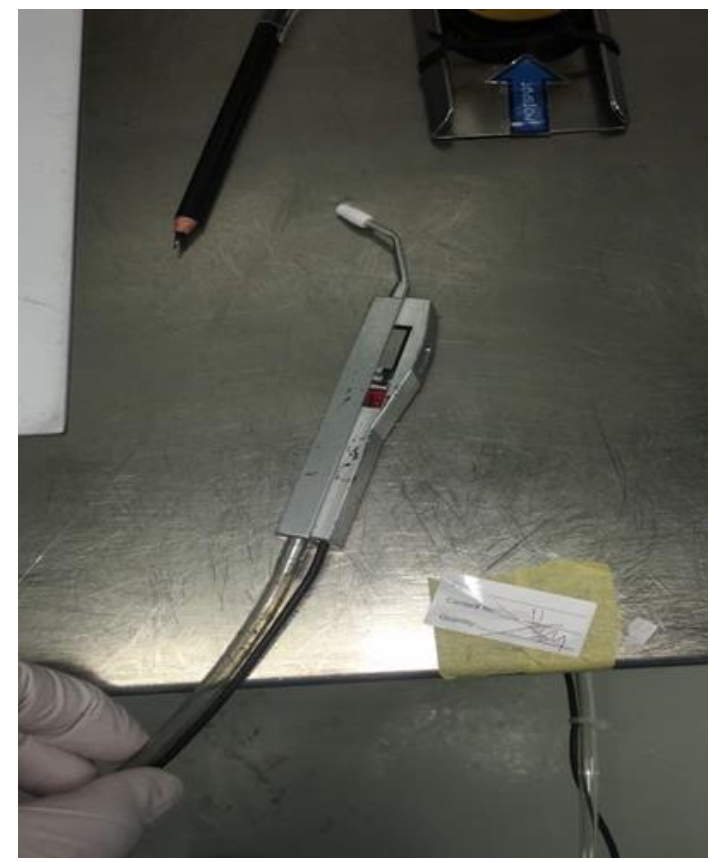

Figure 4. Vacuum Sucker

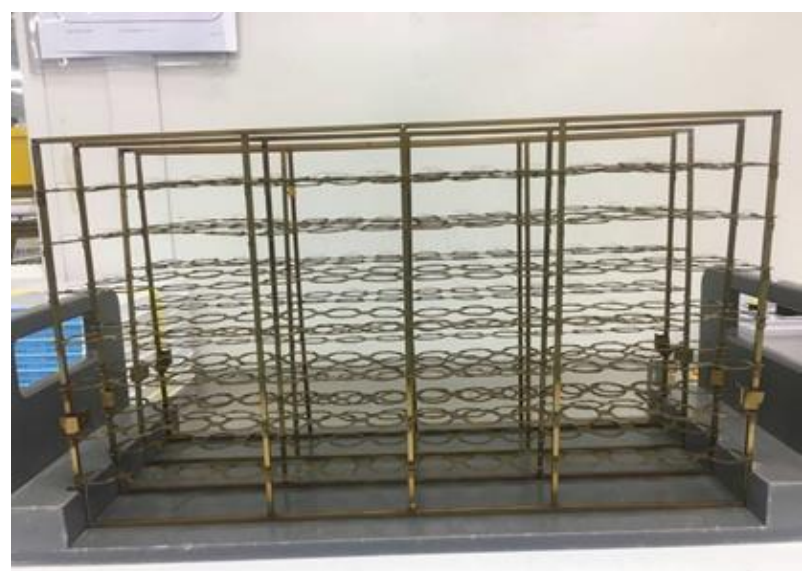

Figure 5. Cassette

\subsection{Software Interface}

Arduino IDE is the software for reading and writes the instruction to the Arduino Uno microcontroller inside it. The microcontroller contains about 7 analog signals and 13 input and output digital which capability to support more data. Solidworks 2017 software is used to build 3D design solution for rapid creation of parts, assemblies, and $2 \mathrm{D}$ drawings.

\section{RESULT AND DISCUSSION}

In this section, the result of the project will be presented and explained in detail. The results include the preliminary design, fabrication, and analysis of the system.

\subsection{Preliminary Design}

Lens Pick and Place robot are designed. The parts which are designed include the cassette, tray, vacuum sucking gripper which is designed to pick the lens and mounted at the end-effector of the machine. The vacuum sucking gripper design contains multiple vacuum sucker attached with silicon to avoid lens deformation. This machine is capable of picking multiple lenses at a time from cassette and place at tray safely.

The inputs and outputs of the Lens Pick and Place robot are identified. The proximity sensor is the one and only input for this project. Besides that, stepper motor and servo motor are the outputs to execute the task. Identifying inputs and outputs of a project need be done. Figure 6 shows the primary result of mechanical structure design of Lens Pick and Place Robot.

As shown in Figure 6, this conceptual design of the robot includes design of nozzle head structure implemented with multiple vacuum suckers are attached with silicon and connected to pump motor. Silicon is attached to reduce damage on the surface of contact lenses. 


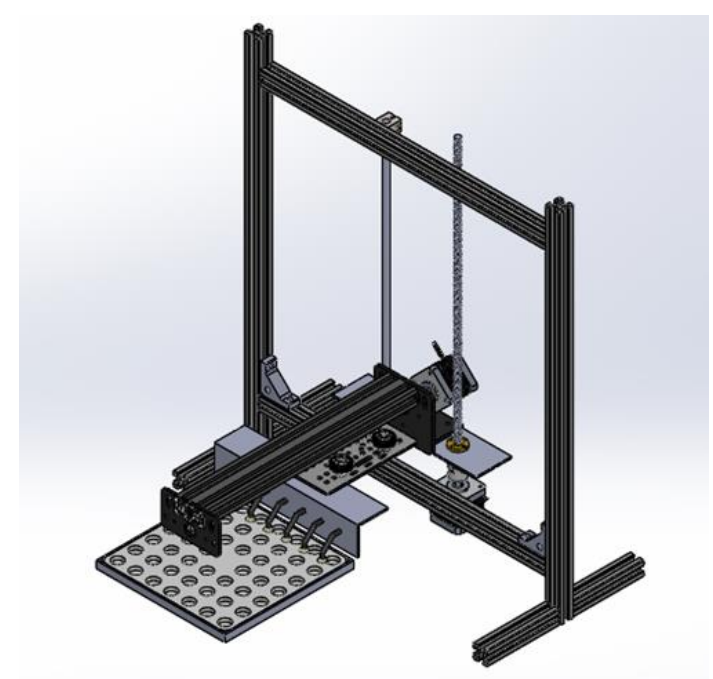

Figure 6. Mechanical design Lens Pick and Place Robot

\subsection{Fabrication}

During fabrication, there are several tools used such as cutting tools and 3D printer to produce mechanism shape. For assembly, this parts must be according to the defined measurement in order to avoid problems in the next step of fabrication and to make sure mechanism structure is stable and flexible design. For electrical hardware, two stepper motors are implemented to make the machine move in 2-axis, and a vacuum pump used for lens sucking and blowing process. Each motor has their own motor driver and connected to Arduino Uno and power supply $12 \mathrm{~V}$ and $10 \mathrm{~A}$ to make sure the source supply is enough for this robot.

This machine also implements the limit switch to limit the movement of the robot and improve the accuracy of a robot. The connection of the electric component is located carefully to make sure the connection between input and output is correct. The size of the robot is $40 \mathrm{~cm}$ length, 50 $\mathrm{cm}$ high and $40 \mathrm{~cm}$ wide. The weight of this machine around $7 \mathrm{Kg}$. The electrical and electronic components are connected and attached well to the robot and controller ready to run with coding.

\subsection{Results}

This robot is programmed to operate automatically, to replace the current manual operation of lens pick and place in Alcon. As compared with the manual process, this robot is much better because it can operate automatically without an operator to run the process. If this robot is implemented to automate the process in the manufacturing area, it will reduce cycle time, manpower and increase lens production hygiene.

For the tested result, the time taken for this machine to complete the pick and place process for one set of trays of contact lenses is 50.9 seconds. This test was taken three times, and the reading shows the same value as the first test. Therefore, this machine proof to be reliable and accurate. Time comparison between machine work and human work is reduced to more than 50 percent, as a time taken for the manual process to complete one set of trays of contact lenses is 60 seconds. Thus, this machine can reduce the processing time of this process when implementing the multiple picks up the nozzle and highspeed motors to this robot. The robot is also highly precision as the stepper motor can provide the movement at the step angle up to 1.8 degrees. Finally, the designed system is more flexible. It can easily be modified or can be customized according to task, space, and process.

\section{CONCLUSION}

The 2 degree of freedoms the robotic arm is able to replace the human workers if the robotic arm is installed properly. As a conclusion, this project can provide some assistance to Alcon Sdn Bhd to automate their manufacturing process. This project provides analytical skills training, hardware assembly training, program writing training and circuitry design training. This project had achieved the goal to provide the best technic that can handle a lot of soft contact lenses while operating automatically, smoothly and providing the flexible design. Besides that, this machine is still under development for application in large-scale manufacturing, change it with more advanced technology in order to make the machine more accurate, move in high speed and can avoid any defect using the latest sensor.

\section{ACKNOWLEDGMENT}

We take it a deemed privilege to express my sincere thanks to Alcon Sdn Bhd and Universiti Teknologi Malaysia for the financial support to complete this project.

\section{REFERENCES}

[1] Borkar, V. (2017). Development of Pick and Place Robot for Industrial. International Research Journal of Engineering and Technology(IRJET), 4(9), 347356.

[2] Sung Hyun Han, Division of Mechanical Engineering and Automation, Kyungnam University Man Hyung Lee, School of Mechanical Engineering, Pusan National University, Hideki Hashimoto, Institute of Industrial Science, University of Tokyo. Japan "Image-Based Visual Servoing Control of a SCARA Robot" KSME International Journal. Vol /4, No. 7, pp. 782-788. 2000.

[3] Alcon (2017). Who We Are: Enhancing sight to improve life. Alcon. Retrieved 23 November 2017 from www.alcon.com.

[4] Montague Ruben (1978). Soft Contact Lenses: Clinical and Applied Technology (1st edition). University of Michigan: Jon Wiley \& Son.

[5] NM Farandos; AK Yetisen; MJ Monteiro; CR Lowe; SH Yun (2014). "Contact Lens Sensors in Ocular Diagnostics". Advanced Healthcare Materials. 4 (6): 792-810. doi:10.1002/adhm.201400504. PMID 25400274.

[6] Andhare, P., \& Rawat, S. (2017). Pick and place industrial robot controller with computer vision. Proceedings - 2nd International Conference on Computing, Communication, Control and Automation, ICCUBEA 2016.

[7] Dömel, A., Kriegel, S., Brucker, M., \& Suppa, M. (2015). Autonomous Pick and Place Operations in Industrial Production. Urai, 39(2), 2391. 
[8] Shariatee, M., Akbarzadeh, A., Mousavi, A., \& Alimardani, S. (2014). Design of an economical SCARA robot for industrial applications. 2014 2nd RSI/ISM International Conference on Robotics and Mechatronics, ICRoM 2014, 534-539

[9] Mousavi, A., Akbarzadeh, A., Shariatee, M., \& Alimardani, S. (2015). Design and construction of a linear-rotary joint for robotics applications. International Conference on Robotics and Mechatronics, ICROM 2015, 229-233

[10] Moghaddam, M., \& Nof, S. Y. (2016). Parallelism of Pick-and-Place operations by multi-gripper robotic arms. Robotics and Computer-Integrated Manufacturing, 42, 135-146.

[11] Karako, Y., Moriya, T., Abe, M., Shimakawa, H., \& Shirahori, S. (2017). A Practical Simulation Method for Pick-and-Place with Vacuum Gripper.

[12] James Schychuck. 7 Depot St. Penn. Pa. 15675 "Pick and place machine" U.S Patent US005649804A issue Jul. 22, 1997.
[13] "IRB 360 FlexPicker" [Online]. Available: http://new.abb.com/products/robotics/ industrialrobots/irb-360 [Accessed: 29 November 2017].

[14] "EPSON SCARA LS3-401S WITH RC90 CONTROLLER" [Online]. Available: https://www.epson.de/en/products/robot/epsonscara-ls3-401s-with-rc90-controller [Accessed: 29 November 2017].

[15] "Control DC and Stepper Motors With L298N Dual Motor Controller Modules and Arduino" [Online]. Available: http://www.instructables.com/id/ControlDC-and-stepper-motors-with-L298N-Dual-Moto/ [Accessed: 24 December 2017].

[16] R.V. Sharan, G.C. Onwubolu (2013). Simulating the Arm Movements of a Stepper Motor Controlled Pick-and Place Robot Using the Stepper Motor Model. International Journal of Advanced Science and Technology. 60, 59-66. 